\title{
The International Neonatal Consortium: collaborating to advance regulatory science for neonates
}

\author{
Mark A. Turner', Jonathan M. Davis ${ }^{2}$, Susan McCune ${ }^{3}$, Ralph Bax, Ronald J Portman ${ }^{5}$, Lynn D. Hudson ${ }^{6}$; the International \\ Neonatal Consortium (INC)
}

A $s$ defined by the US Food and Drug Administration (FDA), regulatory science is the "science of developing new tools, standards and approaches to assess the safety, efficacy, quality and performance of all FDA-regulated products" (1). Nowhere is the need greatest for such standardized methods and new approaches than for the regulation of medicines for neonates (2). Recognizing that researchers often generate results that may influence regulatory decision making, yet are not steeped in the rigorous scientific requirements for regulatory review, the Innovative Medicines Initiative recently released a guidance tool for researchers about how to access support from regulators $(3,4)$.

McCune and Mulugeta called for collaboration in the neonatal community to add clarity, rigor, and predictability to the regulatory path during the development of medicines for the market and, more generally, to efforts that optimize the use of existing and new medicines on the market (5). This can be done with: (i) standardized methods, (ii) standardsof-care agreed upon within the neonatal community, (iii) streamlined approaches to working between multiple sites and/or multiple medicines for a given disease/condition, (iv) innovative trial designs, (v) decision criteria for conducting clinical trials of new therapies (e.g., when should studies in neonates be initiated and what information is needed to design neonatal studies?), (vi) drug development tools qualified by the regulatory agencies for a specific context of use, (vii) clinical outcome assessments, and (viii) modeling approaches such as physiologically based pharmacokinetic and disease progression models, as well as clinical trial simulation tools (3-6). Also important is a shared understanding of formulations for neonates that reflects the practicalities of administration. To date there has been no forum for the neonatal community to develop evidence-based agreements about these approaches to improving the development of drugs for neonates. Because all clinical studies must be informed by the needs and views of people affected by the research, the neonatal collaboration must include a voice for families who have experienced a sick or premature baby.

Outcomes need to be meaningful to families and clinicians or be surrogates that predict these meaningful outcomes. Activities could reflect nonclinical disease models and juvenile animal studies when appropriate.

\section{THE WAY FORWARD: THE INTERNATIONAL NEONATAL CONSORTIUM}

The International Neonatal Consortium (INC) was established in May 2015 in order to provide a global approach to addressing these issues. INC is organized by the nonprofit Critical Path Institute (C-Path), which has 12 consortia working in a "precompetitive space" to share data and expertise to advance regulatory science. Close relationships with regulators in the United States, Europe, Canada, and Japan are central to the work of C-Path (7). Key to the success of C-Path consortia is dedicated project management to convene workshops and drive the production of deliverables, which include qualified biomarkers and clinical outcome assessments, regulatory endorsed clinical trial simulation tools, data standards, and clinical trial databases. Dr. Janet Woodcock, Director of FDA's Center for Drug Evaluation and Research has said: "By uniting stakeholders from research institutions, drug developers, regulatory agencies, patient advocacy and other organizations, INC can develop practical tools that can be incorporated into clinical trials for neonates, which will then lead to more successful, efficient trials and provide this population with better treatments" (8).

A prelaunch meeting in October 2014 at the FDA identified many issues specific to neonates $(6,8)$. INC's inaugural workshop in May 2015 at the European Medicines Agency included a wide range of stakeholders from industry, regulatory agencies, funding bodies (government, private foundations), members of the academic community, representatives of nursing associations, professional societies, and patient advocacy groups who established the initial priorities for the INC. Breakout groups identified critical needs for the therapeutic areas most commonly encountered in the neonatal intensive care unit that contributed

\footnotetext{
IInstitute of Translational Medicine, University of Liverpool, Liverpool, UK; ${ }^{2}$ Department of Pediatrics, Floating Hospital for Children, Tufts Medical Center, and Tufts Clinical and Translational Science Institute, Tufts University, Boston, Massachusetts; ${ }^{3}$ Office of Translational Sciences, Center for Drug Evaluation and Research, U.S. Food and Drug Administration, Silver Spring, Maryland; ${ }^{4}$ Paediatric Medicines, Product Development \& Scientific Support Department, European Medicines Agency, London, UK; ${ }^{5}$ Pediatric Therapeutic Area, Novartis Pharmaceuticals Corporation, East Hanover, New Jersey; ${ }^{6}$ Critical Path Institute, Tucson, Arizona. Correspondence: Mark A. Turner (mark.turner@liverpool.ac.uk) Received 7 February 2016; accepted 15 April 2016; advance online publication 6 July 2016. doi:10.1038/pr.2016.119
} 
most strongly to adverse neonatal outcomes. From these needs assessments, an initial set of INC priorities were selected. Two of the priorities focused on therapeutic areas and two priorities were cross-cutting projects expected to benefit all therapeutic areas.

\section{Treatment of Seizures}

Neonatal seizures are a common problem and a potential target for efforts to improve regulatory science because both short- and long-term outcomes are important and can be assessed; standard-setting for study assessments is plausible on the basis of the state-of-the art; and age-specific studies are required because ontogeny has important effects on pathophysiology, drug targets, and drug disposition. The first deliverable is a master protocol for clinical trials for treating seizures.

\section{Prevention of Bronchopulmonary Dysplasia}

Bronchopulmonary dysplasia continues to be an important adverse consequence of preterm birth with few effective therapeutic approaches for prevention or treatment. The study and management of bronchopulmonary dysplasia would benefit from standardized definitions for symptoms, covariates, and outcomes as well as harmonized data capture between countries for analyzing data, particularly after discharge from the hospital.

\section{Developing a Clinical Pharmacology Position Paper for Neonates That Can be Used to Inform Regulatory Agencies}

The improved understanding of drug evaluation in children over the past decade has been accompanied by evolving regulatory guidance, including neonates and taking account of organ immaturity (4). There is, however, an urgent need to develop a more unified and consistent approach to studying medicines in this subpopulation. Sharing and enhancing the increasing experience with the design and conduct of clinical trials in neonates (e.g., optimal outcome measures, the use of existing data (e.g., when to use extrapolation), and pharmacokinetic studies (e.g., when to use opportunistic assessment of already obtained blood samples, sample sizes for population pharmacokinetic studies)) is a key goal for INC.

\section{Moving toward harmonized data collection}

Critically ill neonates can have life-long illnesses that cause significant burdens for individuals, families, societies, and health care systems. The relatively small number of neonates with primarily rare diseases and the large number of covariates require data to be pooled across many populations and countries. Pooling data on a global scale will require shared definitions of data and databases that can be queried consistently (i.e., interoperable databases). Federating databases that contain routinely collected data will provide useful information on the natural history of disease, biomarkers, clinical end points, standards-of-care, normal laboratory values, and long-term follow-up of outcomes.

\section{INVOLVEMENT OF KEY PARTNERS IN NEONATAL REGULATORY SCIENCE}

INC will develop approaches that integrate neonatal nurses and families, and other interested stakeholders into the design and implementation of clinical studies. INC will work with the European Medicines Agency, FDA, and other regulatory agencies and take account of the roles and needs of industry and other sponsors as well as academic researchers and clinical neonatologists. Meaningful involvement of multiple key stakeholders is needed with approaches that are standardized as much as possible across many populations and cultures.

\section{SUMMARY}

Advancing neonatal regulatory science is essential for the timely development of new treatments and for improved use of existing medicines for neonates. The enormous challenges to conducting clinical studies in neonates who often have a variety of rare diseases can only be addressed through concerted and coordinated efforts of all stakeholders (9). INC will advance regulatory science by focusing on the needs of the neonate and will add value by increasing the predictability of the regulatory path for neonatal medicines, adding an evidence base to enhance prevention and treatment strategies, and demonstrating approaches that are transferable between therapeutic areas.

\section{DISCLAIMER}

The views expressed in this article are the personal views of the authors and may not be understood or quoted as being made on behalf of or reflecting the position of the European Medicines Agency (EMA) or one of its committees or working parties or the position of the U.S. FDA.

\section{ACKNOWLEDGMENTS}

The authors gratefully acknowledge the review by Karel Allegaert, Heike Rabe, Mary Short, and Robin Steinhorn. For more information on the International Neonatal Consortium (INC), see: http://c-path.org/programs/inc/. For those interested in participating in INC workgroups, please contact L.H. (LHudson@c-path.org).

\section{STATEMENT OF FINANCIAL SUPPORT}

The International Neonatal Consortium (INC) is supported in part by grant number U18FD005320-01 from the U.S. Food and Drug Administration (FDA) to the Critical Path Institute (http://c-path.org) and through annual dues of member companies.

Disclosure: The authors declared no potential conflicts of interest with respect to the authorship and/or publication of this article. R.P. is an employee of a Novartis Pharmaceuticals Corporation, East Hanover, NJ, USA. No products are discussed in this article. The consortium aims to improve methods that can be applied to evaluating the safety and effectiveness of any medical product for neonates and is consequently drug agnostic.

\section{REFERENCES}

1. US Food and Drug Administration. Advancing Regulatory Science, 2016. (http://www.fda.gov/ScienceResearch/SpecialTopics/RegulatoryScience/ default.htm) Accessed 21 November 2015.

2. Turner MA. Neonatal drug development. Early Hum Dev 2011;87: 763-8.

3. Innovative Medicines Initiative. Raising Awareness of Regulatory Requirements: A Guidance Tool for Researchers, 2015. (http://www.imi.europa. eu/sites/default/files/uploads/documents/RegulatoryRequirementsGuide. pdf). Accessed 8 July 2015. 


\section{Special Article}

4. European Medicines Agency, Scientific Guidelines: Paediatrics, 2016. (http://www.ema.europa.eu/ema/index.jsp?curl=pages/regulation/ general/general_content_000404.jsp\&mid=WC0b01ac0580926186). Accessed 21 November 2015.

5. McCune SK, Mulugeta YA. Regulatory science needs for neonates: a call for neonatal community collaboration and innovation. Front Pediatr 2014;2:135.

6. Offringa M, Davis JM, Turner MA, et al. Applying Regulatory Science to Develop Safe and Effective Medicines for Neonates: Report of the US Food and Drug Administration First Annual Neonatal Scientific Workshop, 28-29 October 2014. Therapeutic Innovation \& Regulatory Science, 2015. doi: 10.1177/2168479015597730.

7. Brumfield $M$. The Critical Path Institute: transforming competitors into collaborators. Nat Rev Drug Discov 2014;13:785-6.

8. Woodcock J. Quote on the Launch of INC. Tucson, AZ, 2015. (http:// c-path.org/programs/inc/).

9. Davis JM, Turner MA. Global collaboration to develop new and existing drugs for neonates. JAMA Pediatr 2015;169:887-8. 